\title{
Temporal Trends of Hospitalized Patients with Heart Failure in Korea
}

\author{
Jong-Chan Youn, MD, Seongwoo Han, MD, and Kyu-Hyung Ryu, MD \\ Division of Cardiology, Dongtan Sacred Heart Hospital, Hallym University College of Medicine, Hwaseong, Korea
}

\begin{abstract}
Heart failure (HF) is an important cardiovascular disease because of its increasing prevalence, significant morbidity, high mortality and rapidly expanding health care costs. The number of HF patients is increasing worldwide and Korea is no exception. Temporal trends of four representative Korean hospitalized HF registries-the Hallym HF study, the Korean Multicenter HF study, the Korean Heart Failure (KorHF) registry and the Korean Acute Heart Failure (KorAHF) registry showed mild survival improvement reflecting overall HF patient care development in Korea despite the increased severity of enrolled patients with higher incidence of multiple comorbidities. Moreover, device therapies such as implantable cardioverter defibrillator and cardiac resynchronization therapy and definitive treatment such as heart transplantation have been increasing in Korea as well. To prevent HF burden increase, it is essential to set up long term effective prevention strategies for better control of ischemic heart disease, hypertension and diabetes, which might be risk factors for HF development. Moreover, proper HF guidelines, performance measures, and performance improvement programs might be necessary to limit HF burden as well. (Korean Circ J 2017;47(1):16-24)
\end{abstract}

KEY WORDS: Heart failure; Hospitalization; Trends; Korea.

\section{Introduction}

Heart failure (HF) is a clinical syndrome characterized by typical symptoms and signs caused by structural and/or functional cardiac abnormalities." HF is an important cardiovascular disease due to its increasing prevalence, significant morbidity, high mortality and rapidly expanding health care costs. An HF diagnosis has previously been described as more 'malignant' than cancer, given the comparatively low 5-year survival rates. ${ }^{23)}$ In 1991, 5-year mortality from $\mathrm{HF}$ was $11 \%$ higher than the corresponding rates for gastrointestinal cancers. A systematic review found comparative 5-year age-adjusted and gender-adjusted survival rates for cancer,

Received: December 5, 2016

Revised: December 8, 2016

Accepted: December 15, 2016

Correspondence: Seongwoo Han, MD, Division of Cardiology, Dongtan

Sacred Heart Hospital, Hallym University College of Medicine,

Keunjaebong-gil 7, Hwaseong 18450, Korea

Tel: 82-31-8086-2530, Fax: 82-31-8086-2559

E-mail:hansw29@hallym.or.kr

- The authors have no financial conflicts of interest.

This is an Open Access article distributed under the terms of the Creative Commons Attribution Non-Commercial License (http://creativecommons. org/licenses/by-nc/3.0) which permits unrestricted non-commercial use, distribution, and reproduction in any medium, provided the original work is properly cited. stroke, and HF to be generally similar, with observed improvements in survival for all three diseases over the past decade. ${ }^{3 / 4)}$

The prevalence of HF is approximately $1-2 \%$ in the adult population in developed countries, rising to $\geq 10 \%$ among people $>70$ years of age.") On the basis of data from the National Health and Nutrition Examination Survey (NHANES) 2009-2012, about 5.7 million (2.2\%) people over the age of 20 years have HF and more than 550000 people are newly diagnosed with HF every year in the USA. ${ }^{5)}$ By 2030, the prevalence of HF will increase $46 \%$, resulting in more than 8 million people, and total medical costs for HF patients are expected to increase from $\$ 20.9$ billion to $\$ 53.1$ billion. ${ }^{56)}$ In Japan, it is estimated that 1.0 million individuals have $H F$, and the number of Japanese outpatients with left ventricular (LV) dysfunction is predicted to gradually increase from 979000 in 2005 to 1.3 million by 2030.7) In Korea, the prevalence of HF increased approximately two times from $0.75 \%$ in 2002 to $1.53 \%$ in 2013 , and is expected to increase by 2-fold again, from 1.60\% in 2015 to 3.35\% in 2040, when more than 1.7 million Koreans are expected to have $\mathrm{HF}^{\left.8{ }^{89}\right)}$

\section{Korean hospitalized heart failure registry}

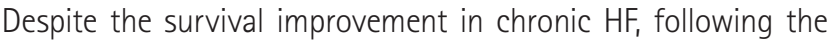
introduction of disease-modifying treatments in the 1990s, 
hospitalization for acute heart failure (AHF) continues to increase in many countries. ${ }^{10-14)} \mathrm{AHF}$ refers to rapid HF onset or worsening of symptoms and/or signs. This is a life-threatening medical condition requiring urgent evaluation and treatment, typically leading to hospital admission.) Estimates of the clinical characteristics, treatment, prognosis and temporal trends of HF hospitalization are central to our understanding of health-care utilization and to efforts to improve patient care and reduce health care costs. Therefore we aimed to investigate the temporal trends of four representative Korean hospitalized HF registries-the Hallym HF study, the Korean Multicenter HF study, the KorHF registry and the KorAHF registry. The Hallym HF study registry was a single university medical center, 4 affiliated hospital-based, retrospective cohort study of Korean hospitalized HF patients, between January 1987 and December $1997(n=1657) .{ }^{15)}$ The Korean Multicenter HF study registry was a nationwide, prospective, observational, multicenter registry from 9 university hospitals for hospitalized Korean HF patients, between January 1998 and August 2003 (n=1759). ${ }^{16)}$ The KorHF registry was a nationwide, prospective, observational, multicenter registry from 24 academic hospitals for hospitalized Korean patients with AHF, between June 2004 and April 2009 ( $n=3200)$. $^{17)}$ The KorAHF registry was also a nationwide, prospective, observational, multicenter cohort registry from 10 tertiary university hospitals for hospitalized Korean patients with AHF between March 2011 and March 2014 $(n=5625){ }^{18)}$ Concomitant with the progress of these four Korean HF registries, the cornerstone of the Korean Society of Heart Failure (KSHF) has been laid firmly with various publications. ${ }^{15-31)}$ Since 2003, KSHF has been Korea's leading academic organization dedicated to the study of heart failure. KSHF has promoted all aspects of research into heart failure, offered education for healthcare professionals, and provided forums where the expertise and experiences in heart failure research and treatment could be shared through periodical conferences, congresses, and symposiums.

In this review article we aimed to evaluate temporal trends seen in the four representative Korean HF registries, and focused on patient population characteristics, clinical status on admission, in-hospital management and clinical outcome. Because long term prognosis from the KorAHF registry are not available yet, we analyzed the KorAHF registry data from published interim analysis results $(n=2066){ }^{18)}$ We also briefly review the temporal trend for device therapies in Korea such as implantable cardioverter defibrillators (ICD) and cardiac resynchronization therapy (CRT) as well as definitive treatment such as heart transplantation.

As shown in Table 1, the recent Korean HF registry patients were older, and had a higher incidence of comorbidities including hypertension, diabetes, atrial fibrillation and stroke compared with previous Korean HF registry populations. Ischemic etiology was associated with a higher proportion of hospitalized HF patients, while hypertension as an HF etiology showed wide variation between different registries. Patients with more severe symptoms were enrolled in the recent registry and more patients underwent echocardiography during the index admission period. While the mean value of hemoglobin, serum sodium and creatinine were comparable between the KorHF and KorAHF registries, the proportion of patients with anemia, hyponatremia and azotemia increased (data not shown). Regarding in-hospital management, more frequent use of intravenous diuretics, inotropes, vasodilators, mechanical ventilation and renal replacement therapy were noted in the recent period, reflecting increased HF severity in the patient population. The prescription rate for beta-blockers at discharge was lower in the KorAHF registry while that for angiotensin converting enzyme inhibitor (ACEi)/angiotensin receptor blocker (ARB) was higher. Despite increased HF severity in enrolled patients, the recent registry showed mild improvement of in-hospital mortality indicating the overall development of hospitalized HF patient care in Korea.

\section{Age}

One of the outstanding findings in the comparisons between the four Korean hospitalized HF registries is the rapidly increasing mean age of the patient population (Fig. 1). HF is one of the major causes of death and hospitalization for elderly patients. The Korean National Health Insurance Service (NHIS) sample cohort data showed that the prevalence of HF was $12.6 \%$ in subjects aged 80 years or older, while it was $1.0 \%$ and $5.5 \%$ in those aged 40 59 years and 60-79 years, respectively. The prevalence of HF in subjects aged 80 years or older was approximately 8 -fold higher than the overall prevalence of HF (1.53\%). ${ }^{8}$ Age is an important prognostic factor in the Hallym HF registry, the Korean Multicenter HF study registry and KorHF registry as well. ${ }^{15-17)}$ The KorAHF registry also showed that old age ( $\geq 70$ years) was an independent predictor of post-discharge mortality. ${ }^{18)}$ Data from other countries revealed that the overall number of hospitalizations for heart failure has increased, particularly in the aged population. ${ }^{1113)}$ With the aging of the general population, the epidemiologic burden of HF might increase substantially.

\section{Comorbidities}

Temporal trends in hospitalized HF patient comorbidities showed increasing prevalence of hypertension and diabetes (Fig. 2). The Korean 
Table 1. Comparison of four Korean hospitalized HF registry

\begin{tabular}{|c|c|c|c|c|}
\hline & $\begin{array}{l}\text { Hallym HF study } \\
\qquad(n=1657)\end{array}$ & $\begin{array}{l}\text { Korean multicenter HF study } \\
\qquad(n=1759)\end{array}$ & $\begin{array}{c}\text { KorHF } \\
(n=3200)\end{array}$ & $\begin{array}{c}\text { KorAHF } \\
(n=2066)\end{array}$ \\
\hline Time period & January 1987-December 1997 & January 1998-August 2003 & June 2004-April 2009 & March 2011-May 2012 \\
\hline No. of participating hospitals & 4 & 9 & 24 & 10 \\
\hline \multicolumn{5}{|l|}{ Demographics } \\
\hline Male (\%) & 43.2 & 52.9 & 50 & 55 \\
\hline Age (year) & $63.7 \pm 13.4$ & $64.1 \pm 14.3$ & $67.6 \pm 14.3$ & $69 \pm 14$ \\
\hline Hypertension (\%) & 40.4 & 30.2 & 46.5 & 59 \\
\hline Diabetes mellitus (\%) & 19.9 & 25.6 & 30.5 & 36 \\
\hline Atrial fibrillation (\%) & 9.2 & 27.5 & 22.4 & 27 \\
\hline Stroke $(\%)$ & 9.5 & 8.6 & 18.9 & 15 \\
\hline \multicolumn{5}{|l|}{ Etiology $(\%)$} \\
\hline Ischemic & 29.3 & 32.3 & 52.3 & 38 \\
\hline Hypertensive & 26.7 & 16.5 & 36.7 & 6 \\
\hline Cardiomyopathy & 25.5 & 22.7 & 26.5 & 21 \\
\hline Idiopathic dilated & 15.3 & 14.9 & 23.3 & 15 \\
\hline Valvular & 17.2 & 13.5 & 12.7 & 13 \\
\hline \multicolumn{5}{|l|}{ In-hospital management (\%) } \\
\hline Intravenous inotropes & - & - & 35.8 & 40.0 \\
\hline IABP & - & - & 3.3 & 3.9 \\
\hline Mechanical ventilation & - & - & 7.1 & 14.0 \\
\hline Hemodialysis & - & - & 4.1 & 4.5 \\
\hline CRRT & - & - & & 4.3 \\
\hline ECMO/PCPS & - & - & & 2.5 \\
\hline \multicolumn{5}{|l|}{ Medication at discharge (\%) } \\
\hline Beta-blocker & - & - & 58.6 & 44.0 \\
\hline ACEi/ARB & 65.1 (ACEi only) & - & 53.7 & 65.0 \\
\hline Aldosterone antagonist & - & - & 53.1 & 40.4 \\
\hline Digitalis & 52.0 & - & 13.9 & - \\
\hline $\begin{array}{l}\text { Total mean medical cost } \\
\left(\times 10^{3} \mathrm{KRW}\right)\end{array}$ & - & - & 6658 & 7700 \\
\hline Admission duration (day) & $11.3 \pm 12.2$ & - & $9^{*}$ & $8^{*}$ \\
\hline In-hospital mortality (\%) & 5.8 ( 1 month mortality) & - & 6.4 & 5.2 (excluding $H T x$ ) \\
\hline 6-month cumulative survival (\%) & 87.4 & 90.5 & - & 90.8 \\
\hline 1-year cumulative survival (\%) & 82.0 & 80.1 & 85.0 & - \\
\hline
\end{tabular}

*Expressed as median duration. HF: heart failure, KorHF: Korean heart failure, KorAHF: Korean acute heart failure, IABP: intraaortic balloon pump, CRRT: continuous renal replacement therapy, ECMO: extracorporeal membrane oxygenation, PCPS: percutaneous cardiopulmonary support, ACEi: angiotensin converting enzyme-inhibitor, ARB: angiotensin receptor blocker, KRW: Korean won, HTx: heart transplantation

NHIS sample cohort data revealed that hypertension and diabetes were common in HF patients. The prevalence of hypertension as a comorbidity in hospitalized HF patients remained high, while diabetes was markedly increasing in 2013 compared to 2003 (hypertension $46.2 \%$, and diabetes $33.4 \%$ in 2003 ; hypertension $43.6 \%$, and diabetes 49.1\% in 2013, respectively). ${ }^{8}$ ) Data from Western Australia also revealed that hypertension and diabetes were the predominant comorbidities associated with HF and the prevalence of risk factors

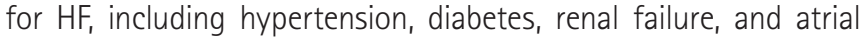
fibrillation, increased between 1990 and 2005, which along with 


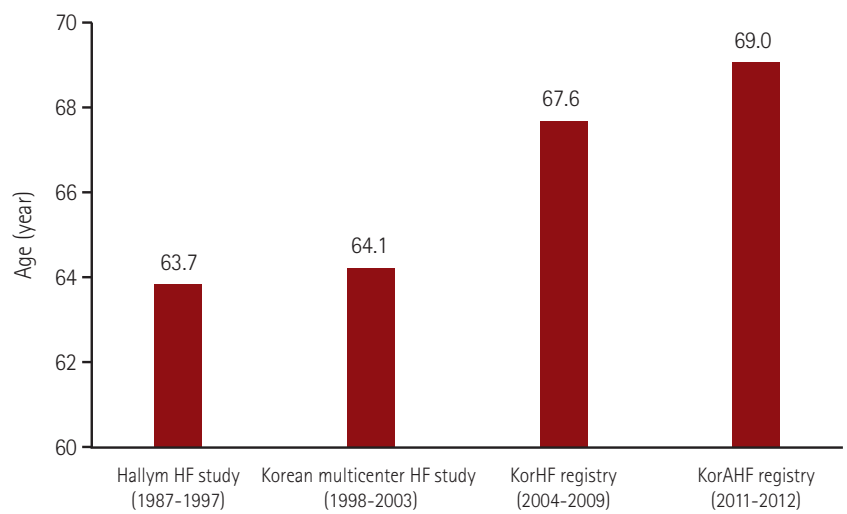

Fig. 1. Temporal trends of mean age in four Korean hospitalized HF registry. HF: Heart failure, KorHF: Korean heart failure, KorAHF: Korean acute heart failure.

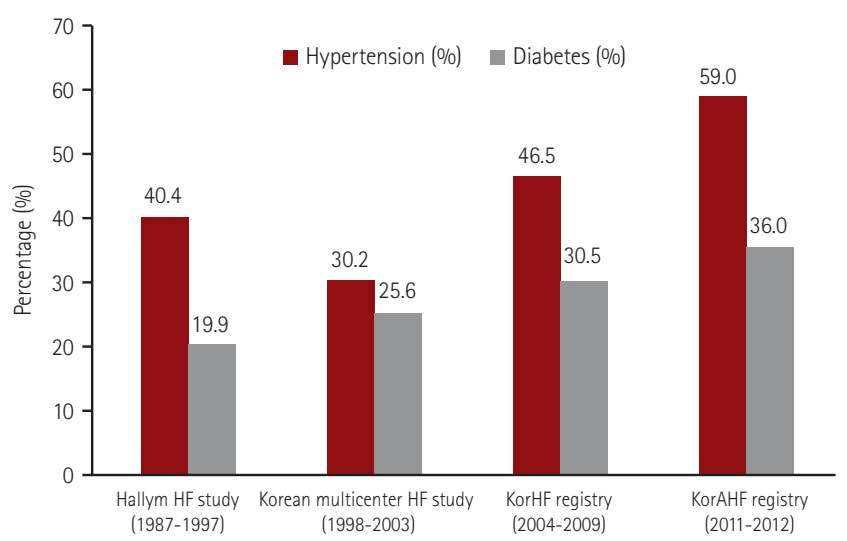

Fig. 2. Temporal trends of comorbidities in four Korean hospitalized HF registry. HF: heart failure, KorHF: Korean heart failure, KorAHF: Korean acute heart failure.

aging population demographics provided a note of caution with respect to future population trends for HF."11

\section{Heart failure etiologies}

Ischemic etiology is responsible for a high proportion of hospitalized HF cases, suggesting the need for better control of ischemic heart disease in order to reduce HF hospitalization (Fig. 3). The number of ischemic heart disease patients has increased due to the accelerated westernization of lifestyle in Korean people, and the number of survivors of an acute coronary syndrome has dramatically increased due to the development of interventional and medical progress in the general cardiovascular treatment. Recently, similar trends showing ischemia as the chief cause of HF were observed in

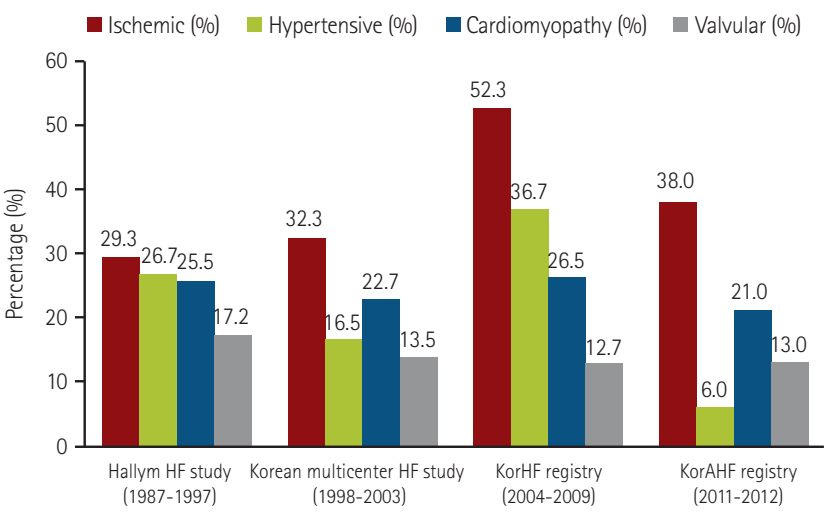

Fig. 3. Temporal trends of etiologies in four Korean hospitalized HF registry. HF: heart failure, KorHF: Korean heart failure, KorAHF: Korean acute heart failure.

Asia, including China and Japan. ${ }^{7 / 8)}$

Cardiomyopathy and valvular heart disease etiologies underlying HF hospitalization showed consistent proportions during different time periods, while hypertension as an HF etiology showed wide variation between different registries. Hypertension as an HF etiology was significantly lower in the KorAHF registry compared to the other registries. This may result from different initial evaluation protocols in the KorAHF registry or the limited number of participating tertiary hospitals with higher rates of hypertension awareness, diagnosis and control. ${ }^{18)}$ It is sometimes difficult to differentiate whether hypertension is merely an accompanying disease or an HF hospitalization etiology. It is necessary to more clearly define 'hypertensive heart failure' in which uncontrolled hypertension at clinical presentation might resultin decompensation in the absence of other identifiable causes.

\section{Clinical outcomes and prognostic factors}

Even though the time point for survival analysis showed variation between the different registries, clinical outcomes showed mild improvement despite the increased HF severity in enrolled patients, reflecting the overall development of $\mathrm{HF}$ patient care in Korea (Fig. 4). In-hospital mortality was $5-8 \%$, which is comparable to other registries. The cumulative Kaplan-Meier estimates for survival using 3 available previous registry data sets showed modest improvement in long term survival (Fig. 5). For the proper analysis of long term clinical outcomes, more detailed analysis such as propensity score matching might be necessary. However, it is common that temporal trends in clinical outcomes show mild or limited improvement irrespective of medical and surgical care 


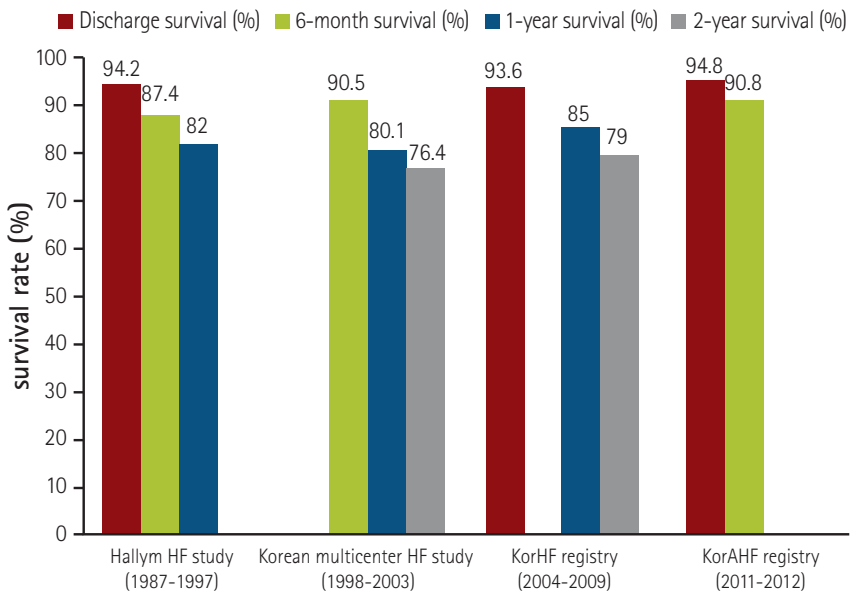

Fig. 4. Temporal trends of clinical outcome in four Korean hospitalized HF registry. HF: heart failure, KorHF: Korean heart failure, KorAHF: Korean acute heart failure.

development for $\mathrm{HF}$, because of the increased HF severity seen in enrolled patients. ${ }^{32 / 33}$ Independent prognostic factors from the four representative Korean hospitalized HF registries are summarized in Table 2.

Hyponatremia was an independent predictor of poor prognosis in the Hallym HF registry, the KorHF registry, the KorAHF registry and other Asian hospitalized HF registries. 15171/18/34/35) Data from the KorHF registry revealed that hyponatremia at admission was associated with higher all-cause mortality, irrespective of whether hyponatremia improved during hospitalization. ${ }^{20)}$ A multivariate regression analysis of the KorAHF registry data also showed that hyponatremia at admission was an independent predictor of inhospital mortality and post-discharge mortality. ${ }^{18)}$ Anemia is a well-known prognostic factor in patients with $\mathrm{HF}$ and is commonly observed in hospitalized HF patients. The KorHF registry showed that anemia on admission was an independent predictor for allcause mortality in hospitalized HF patients..$^{17)}$ Renal dysfunction is

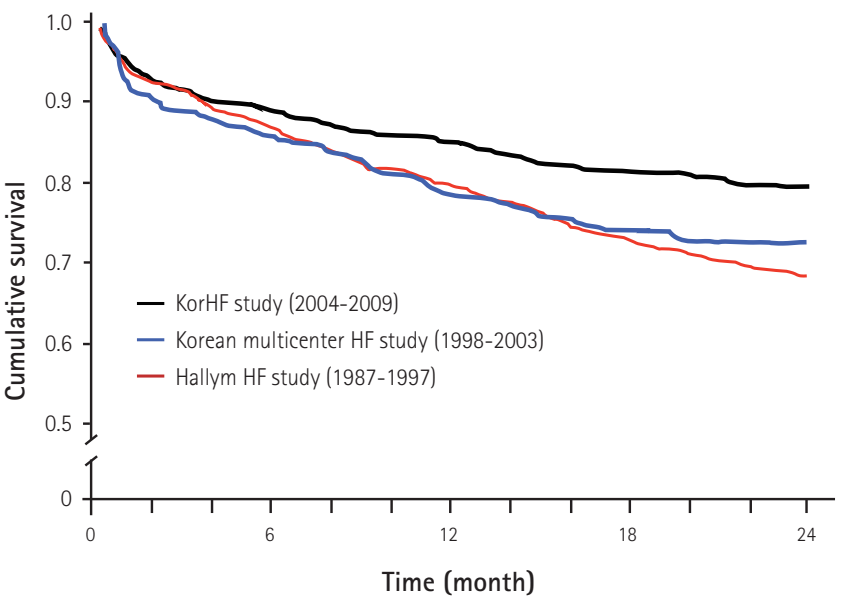

Fig. 5. Cumulative Kaplan-Meier estimates of survival according to different time periods in three Korean hospitalized HF registry. KorHF: Korean heart failure, HF: heart failure.

one of the most important findings during hospitalization and a prognostic factor for hospitalized HF patients. The KorHF registry revealed that renal dysfunction was associated with poor clinical outcomes..$^{17)}$ A multivariate regression analysis of KorAHF registry data also showed that renal dysfunction at admission was one of the most important prognostic factors for in-hospital mortality and post-discharge mortality. ${ }^{18)}$

The cumulative survival curves for HF etiologies are shown in Fig. 6. The Hallym HF study revealed that HF caused by ischemic heart disease was associated with the poorest cumulative survival of $80.5 \%$ at 6 months, $30.2 \%$ at 5 years and $18.4 \%$ at 10 years, while HF caused by hypertensive heart disease predicted better survival with $90.8 \%$ at 6 months, $53.8 \%$ at 5 years and $31.1 \%$ at 10 years. ${ }^{15)}$ These patterns were consistent with a subsequent Korean multicenter HF study as well. HF caused by ischemic heart disease showed the poorest cumulative survival with $79.4 \%$ at 6 months, $70.2 \%$ at 1 year and $59.7 \%$ at 2 years, while $\mathrm{HF}$ caused by valvular

Table 2. Independent predictors of clinical outcome in four Korean hospitalized HF registry

\begin{tabular}{|c|c|c|c|}
\hline $\begin{array}{l}\text { Hallym HF study } \\
(n=1657)\end{array}$ & $\begin{array}{l}\text { Korean multicenter } \\
\text { HF study }(n=1759)\end{array}$ & $\begin{array}{c}\text { KorHF } \\
(n=3200)\end{array}$ & $\begin{array}{c}\text { KorAHF } \\
(n=2066)\end{array}$ \\
\hline Hyponatremia & Previous MI & Old age & Old age \\
\hline Diabetes & Diabetes & Hyponatremia & Hyponatremia \\
\hline Stroke & Stroke & Previous HF & Renal dysfunction \\
\hline Smoking & & Anemia & $\begin{array}{l}\text { HF aggravation due } \\
\text { to renal failure }\end{array}$ \\
\hline \multirow[t]{2}{*}{ Interruption of ACE inhibitors } & & High NT-proBNP & \\
\hline & & Beta-blocker at discharge & \\
\hline
\end{tabular}

HF: heart failure, KorHF: Korean heart failure, KorAHF: Korean acute heart failure, MI: myocardial infarction, ACE: angiotensin converting enzyme, NT-proBNP: N-terminal prohormone of brain natriuretic peptide 

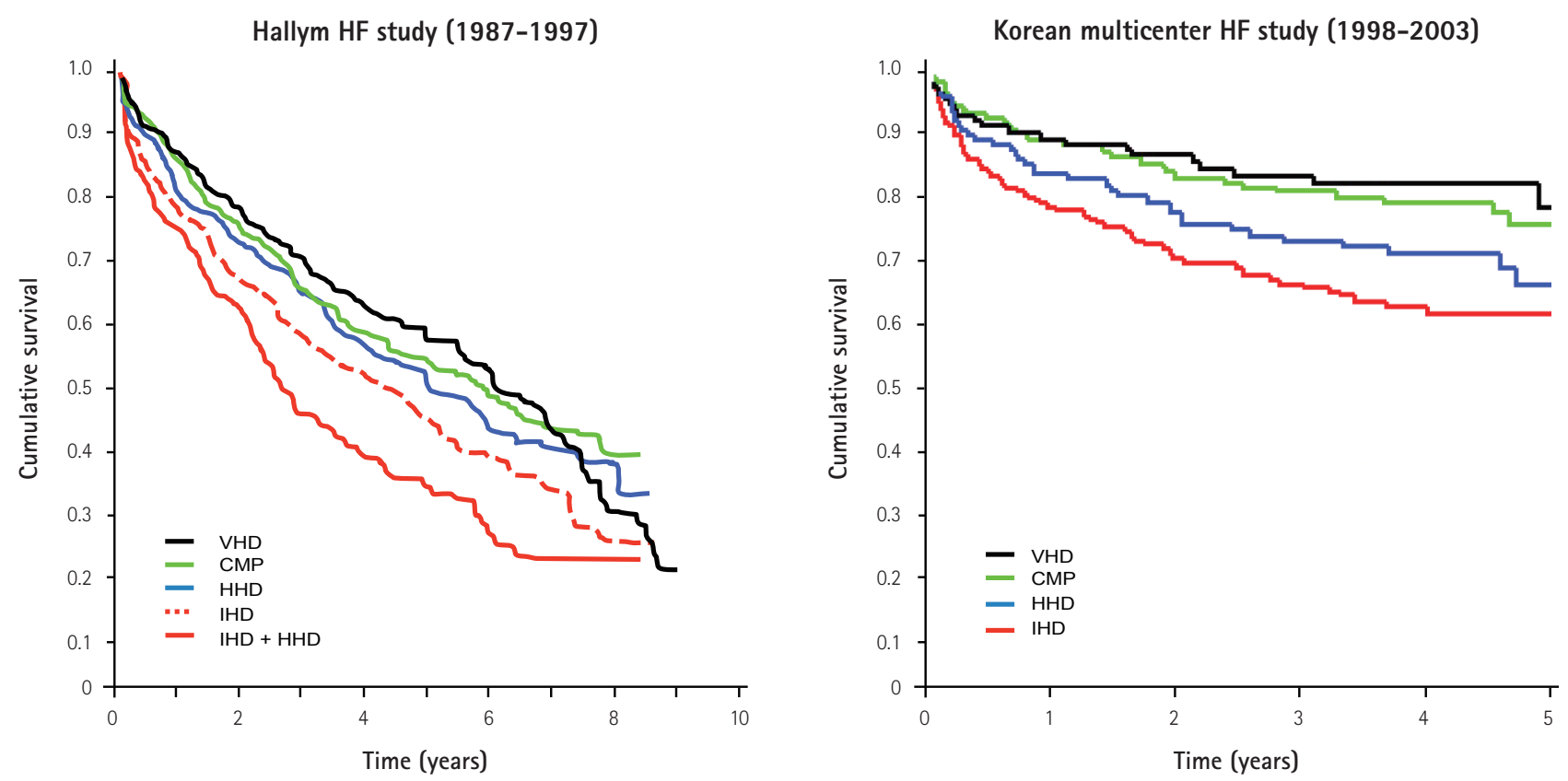

Fig. 6. Cumulative Kaplan-Meier estimates of survival for hospitalized patients with heart failure according to the etiologies. HF: heart failure, VHD: valvular heart disease, CMP: cardiomyopathy, HHD: hypertensive heart disease, IHD: Ischemic heart disease.

heart disease was associated with better survival with $92.6 \%$ at 6 months, $91.0 \%$ at 1 year and $89.2 \%$ at 2 years followed by HF caused by cardiomyopathy and hypertensive heart disease ${ }^{16)}$. However, long term survival of valvular heart disease patients in the Hallym HF study revealed a poor prognosis 7-9 years after index admission requiring close attention to these patients. HF etiology can be highly variable and many patients will have several comorbid pathologies that result in HF hospitalization. Identification of these diverse pathologies should be part of the diagnostic workup, as they may offer specific therapeutic opportunities.")

\section{In-hospital management}

More frequent use of intravenous diuretics, inotropes, vasodilators, mechanical ventilation and renal replacement therapy in the recent time period might result from the fact that patients with more severe symptoms and multiple comorbidities were enrolled in the recent registry. The advance of more active interventional treatment such as mechanical ventilation, renal replacement therapy and percutaneous cardiopulmonary support (PCPS) may be related to the improved in-hospital mortality in AHF patients. PCPS have been actively engaged in the acute care of patients with cardiogenic shock and are associated with clinical outcome improvement in Korea. ${ }^{36)}$

Guideline directed medical therapy and performance measures are important issues in the management of hospitalized HF patients. Interruption of ACEi treatment was an independent prognostic factor in the Hallym HF registry, while beta-blocker prescription at discharge showed better clinical outcomes in the KorHF registry. When 1527 patients from the KorHF registry with left ventricular systolic dysfunction who survived hospitalization were evaluated, multivariate analysis revealed that adherence to ACE inhibitor treatment or ARB use at discharge were significantly associated with mortality and readmission at 60 days, and adherence to betablockers at discharge with mortality at 1 year. ${ }^{22}$

\section{Implantable cardioverter defibrillators and cardiac resynchronization therapy in Korea}

In Korea, device therapy for HF patients such as ICD and CRT has been underutilized because of cultural proscriptions, low $\mathrm{HF}$ awareness of patients and strict insurance coverage criteria. However, device therapy has recently increased steadily as shown in Fig. 7. To get real world data regarding the risks and benefits

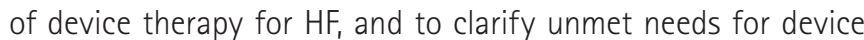
therapy in Korean HF patients, it is necessary to establish a prospective nationwide HF device therapy registry near in the future. 


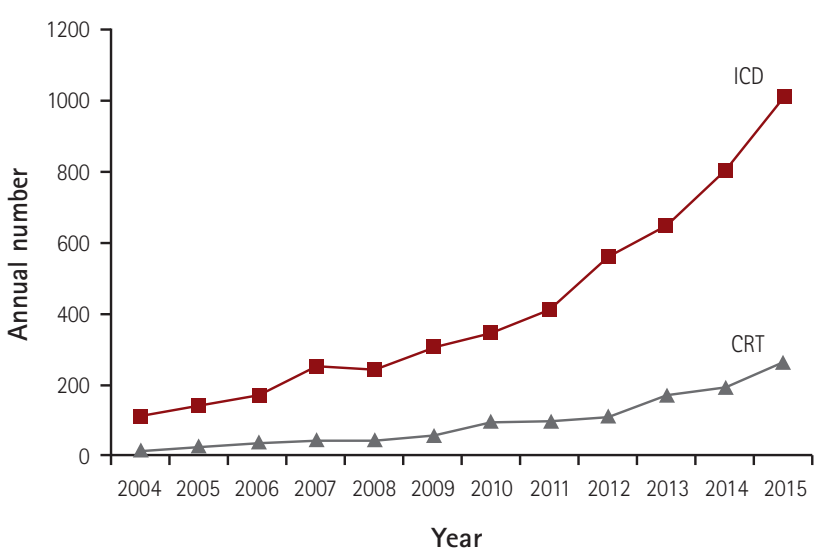

Fig. 7. Temporal trends of implantable cardioverter defibrillator and cardiac resynchronization therapy in Korea . ICD: implantable cardioverter defibrillator, CRT: cardiac resynchronization therapy.

\section{Heart transplantation in Korea}

In Korea, the first heart transplantation was performed at Asan medical center in 1992. The recipient was 51 years old at that time and is still alive after more than 23 years. By December 31st 2015, a total of 1136 heart transplants were done in Korea. ${ }^{37)}$ Before 2000, annual heart transplants were less than 20, however transplants increased to more than 50 by 2007 and recently the annual number was over 120 (Fig. 8). A nationwide heart transplant cohort study under the Korean Organ Transplant Registry (KOTRY) since 2013 has been started. In Korea, unlike other Asian countries, where religious and social beliefs hinder organ donation, the number of heart transplants have increased steadily recent periods. ${ }^{37(38)}$ However there is still a significant organ shortage even though organ donation is increasing in Korea, because there is a rapidly increasing number of end-stage HF patients requiring definitive treatment.

Accordingly, mechanical circulatory support devices, particularly continuous-flow left ventricular assist devices (LVADs) are increasingly seen as an alternative to heart transplantation in North America and Europe. The number of patients with a permanent LVAD who are considered neither too old nor ineligible for transplantation is constantly growing. For a majority of these patients, lifelong LVAD therapy, despite eligibility for transplantation, has become a clinical reality.' However, LVADs are not actively used in Korean end-stage HF patients' care, because of government regulation and insurance issues.

\section{Future strategies to reduce heart failure burden}

The prevalence of hypertension, diabetes and ischemic heart

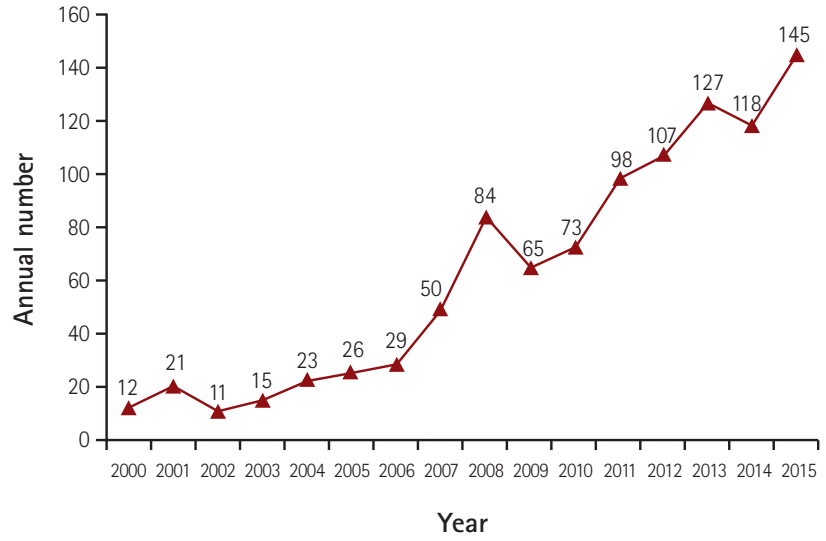

Fig. 8. Temporal trends of heart transplantation in Korea.

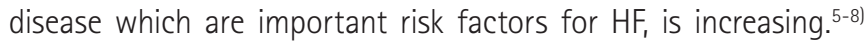
Patients in acute cardiovascular disease status will survive longer due to the development of advanced medical and surgical therapies, as well as accelerated aging of the general population, which will result in more patients at risk for developing HF.6) If increased HF burdens are to be avoided, the prevention of incident HF and proper guidelines for HF treatment are essential. Proper understanding of risk factors for HF is important to the development of preventive strategies. Demographic risk factors for the development of $\mathrm{HF}$ include older age, male gender, smoking, and obesity, while specific diseases such as ischemic heart disease, hypertension and diabetes also contribute significantly to the development of HF.6) Ischemic heart disease is still one of the most important risk factors for hospitalized HF in Korea and requires a special monitoring program. Hypertension is associated with a smaller relative risk for HF development than that associated with ischemic heart disease, but contributes more to overall HF burden because of its higher prevalence. $\left.{ }^{6}\right)$ The prevalence of diabetes as a comorbidity in Korean hospitalized HF patients is steadily increasing and it is well known that diabetes, insulin resistance, and obesity are also linked to HF development. To prevent increased HF burdens, it is essential to set up long term effective pre-emptive prevention strategies for better control of ischemic heart disease, hypertension, and diabetes which are risk factors for HF development.

Not only the prevention, but also the treatment of HF, can be improved through enhanced adherence to guideline directed management.6) In an effort to increase the use of evidence-based prevention and treatment approaches, the KSHF has produced Korean guidelines for the prevention and treatment of chronic HF in 2015, and for AHF in 2016. By facilitating measurements of cardiovascular healthcare quality, performance measures may serve as effective methods to accelerate appropriate translation of 
scientific evidence into clinical practice.) Performance improvement programs have facilitated the implementation of evidence-based therapies in both hospital and ambulatory care settings and it is well known that providing better levels of care based on performance measures are associated with better patient survival rates in patients with HF.622) Therefore, proper guidelines, performance measures, and performance improvement programs can have a substantial impact on cardiovascular prevention and treatment and will be important tools for limiting the burden of $\mathrm{HF}$ as well.

In summary, the temporal trends seen in four representative Korean HF registries-the Hallym HF study, the Korean Multicenter HF study, the KorHF registry and the KorAHF registry, showed mild improvement in $\mathrm{HF}$ mortality reflecting the overall development of AHF patient care in Korea despite the increased disease severity of enrolled patients including higher incidence of multiple comorbidities. To prevent increasing HF burdens, it is essential to set up long term effective pre-emptive prevention strategies for better control of ischemic heart disease, hypertension and diabetes, which might be risk factors for the development of HF. Moreover, proper HF guidelines, performance measures, and performance improvement programs are necessary to limit HF burden as well.

\section{Acknowledgements}

This study was supported by the fund from the Korean Society of Cardiology (201403-03) and by Basic Science Research Program through the National Research Foundation of Korea (NRF) funded by the Ministry of Science, ICT \& Future Planning (NRF2015R1C1A1A02036645). The funders had no role in study design, data collection and analysis, decision to publish, or preparation of the manuscript. The authors have declared that no competing interests exist.

The authors express sincere gratitude to Korean representative heart failure physicians (Myung-Mook Lee, Byung-Hee Oh, Shung Chull Chae, Myeong-Chan Cho, Eun-Seok Jeon, Jae-Joong Kim, Dong-Ju Choi, Seok-Min Kang, and Byung-Su Yoo) who actively participated in the Korean hospitalized heart failure registry study.

\section{References}

1. Ponikowski P, Voors AA, Anker SD, et al. 2016 ESC Guidelines for the diagnosis and treatment of acute and chronic heart failure: The Task Force for the diagnosis and treatment of acute and chronic heart failure of the European Society of Cardiology (ESC) Developed with the special contribution of the Heart Failure Association (HFA) of the
ESC. Eur Heart J 2016;37:2129-200.

2. Stewart S, MacIntyre K, Hole DJ, Capewell S, McMurray JJ. More 'malignant' than cancer? Five-year survival following a first admission for heart failure. Eur J Heart Fail 2001;3:315-22.

3. Ziaeian B, Fonarow GC. Epidemiology and aetiology of heart failure. Nat Rev Cardio/ 2016;13:368-78.

4. Askoxylakis V, Thieke C, Pleger ST, et al. Long-term survival of cancer patients compared to heart failure and stroke: a systematic review. BMC Cancer 2010;10:105.

5. Writing Group M, Mozaffarian D, Benjamin EJ, et al. Heart disease and stroke statistics-2016 update: a report from the American Heart Association. Circulation 2016;133:e38-360.

6. Heidenreich PA, Albert NM, Allen LA, et al. Forecasting the impact of heart failure in the United States: a policy statement from the American Heart Association. Circ Heart Fail 2013;6:606-19.

7. Okura $Y$, Ramadan MM, Ohno Y, et al. Impending epidemic: future projection of heart failure in Japan to the year 2055. Circ J 2008;72:489-91.

8. Lee JH, Lim NK, Cho MC, Park HY. Epidemiology of Heart Failure in Korea: Present and Future. Korean Circ J 2016;46:658-64.

9. Park JJ, Choi DJ. Heart failure epidemic: an expected phenomenon. Korean Circ J 2016;46:610-2.

10. Fang J, Mensah GA, Croft JB, Keenan NL. Heart failure-related hospitalization in the U.S., 1979 to 2004. J Am Coll Cardiol 2008;52:428-34.

11. Teng TH, Finn J, Hobbs M, Hung J. Heart failure: incidence, case fatality, and hospitalization rates in Western Australia between 1990 and 2005. Circ Heart Fail 2010;3:236-43.

12. Filippatos G, Farmakis D, Bistola V, et al. Temporal trends in epidemiology, clinical presentation and management of acute heart failure: results from the Greek cohorts of the Acute Heart Failure Global Registry of Standard Treatment and the European Society of Cardiology-Heart Failure pilot survey. Eur Heart J Acute Cardiovasc Care 2014 [Epub ahead of print]. doi: 10.1177/2048872614527012

13. Agarwal SK, Wruck L, Quibrera M, et al. Temporal Trends in Hospitalization for Acute Decompensated Heart Failure in the United States, 1998-2011. Am J Epidemio/ 2016;183:462-70.

14. Kang SM, Cho MC. Prognostic Factors in Hospitalization for Heart Failure in Asia. Heart Fail Clin 2015;11:543-50.

15. Lee $Y$. Chinical characteristics of korean patients with congestive heart failure. Korean J Med 1998;55:446-52.

16. Han SW, Ryu KH, Chae SC, et al. Multicenter analysis of clinical characteristics and prognostic factors of patients with congestive heart failure in Korea. Korean Circ J 2005;35:357-61.

17. Choi DJ, Han S, Jeon ES, et al. Characteristics, outcomes and predictors of long-term mortality for patients hospitalized for acute heart failure: a report from the korean heart failure registry. Korean 
Circ J 2011;41:363-71.

18. Lee $S E$, Cho HJ, Lee HY, et al. A multicentre cohort study of acute heart failure syndromes in Korea: rationale, design, and interim observations of the Korean Acute Heart Failure (KorAHF) registry. Eur J Heart Fail 2014;16:700-8.

19. Hong SJ, Oh J, Kang SM, et al. Clinical implication of right bundle branch block in hospitalized patients with acute heart failure: data from the Korean Heart Failure (KorHF) Registry. Int J Cardiol 2012;157:416-8.

20. Lee SE, Choi DJ, Yoon CH, et al. Improvement of hyponatraemia during hospitalisation for acute heart failure is not associated with improvement of prognosis: an analysis from the Korean Heart Failure (KorHF) registry. Heart 2012;98:1798-804.

21. Yoon $\mathrm{CH}$, Youn $\mathrm{TJ}$, Ahn $\mathrm{S}$, et al. Low serum total cholesterol level is a surrogate marker, but not a risk factor, for poor outcome in patients hospitalized with acute heart failure: a report from the Korean Heart Failure Registry. J Card Fail 2012;18:194-201.

22. Youn YJ, Yoo BS, Lee JW, et al. Treatment performance measures affect clinical outcomes in patients with acute systolic heart failure: report from the Korean Heart Failure Registry. Circ J 2012;76:1151-8.

23. Oh J, Kang SM, Hong $N$, et al. Hemoconcentration is a good prognostic predictor for clinical outcomes in acute heart failure: data from the Korean Heart Failure (KorHF) Registry. Int J Cardiol 2013;168:4739-43.

24. Oh J, Kang SM, Hong $N$, et al. The CKD-EPI is more accurate in clinical outcome prediction than MDRD equation in acute heart failure: data from the Korean Heart Failure (KorHF) Registry. Int J Cardiol 2013;167:1084-7.

25. Park HS, Kim H, Park JH, et al. QRS prolongation in the prediction of clinical cardiac events in patients with acute heart failure: analysis of data from the Korean Acute Heart Failure Registry. Cardiology 2013;125:96-103.

26. Park SJ, On YK, Byeon K, et al. Short- and long-term outcomes depending on electrical dyssynchrony markers in patients presenting with acute heart failure: clinical implication of the first-degree atrioventricular block and QRS prolongation from the Korean Heart Failure registry. Am Heart J 2013;165:57-64 e2.

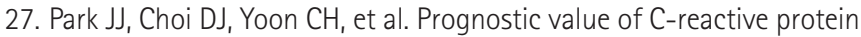

as an inflammatory and $\mathrm{N}$-terminal probrain natriuretic peptide as a neurohumoral marker in acute heart failure (from the Korean Heart Failure registry). Am J Cardio/ 2014;113:511-7.

28. Kang SH, Park JJ, Choi DJ, et al. Prognostic value of NT-proBNP in heart failure with preserved versus reduced EF. Heart 2015;101:1881-8.

29. Oh J, Kang SM, Song MK, et al. Clinical benefit of spironolactone in patients with acute decompensated heart failure and severe renal dysfunction: Data from the Korean Heart Failure Registry. Am Heart J 2015;169:713-20.e3.

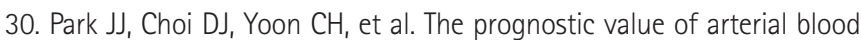
gas analysis in high-risk acute heart failure patients: an analysis of the Korean Heart Failure (KorHF) registry. Eur J Heart Fail 2015;17:601-11.

31. Ryu KH, Han SW. Clinical Characterisrics and prognositic factors of patients with congestive heart failure in Korea (epidemiology of congestive heart failure in Korea). J Korean Soc Hypertens 2002;8:38-47.

32. Youn JC, Seo SM, Lee HS, et al. Trends in hospitalized acute myocardial infarction patients with heart failure in Korea at 1998 and 2008. $J$ Korean Med Sci 2014;29:544-9.

33. Sato N. Epidemiology of Heart Failure in Asia. Heart Fail Clin 2015;11:573-9.

34. Bae MH, Chae SC. Hyponatremia in acute heart failure: a marker of poor condition or a mediator of poor outcome? Korean J Intern Med 2015;30:450-2.

35. Yoo BS, Park JJ, Choi DJ, et al. Prognostic value of hyponatremia in heart failure patients: an analysis of the Clinical Characteristics and Outcomes in the Relation with Serum Sodium Level in Asian Patients Hospitalized for Heart Failure (COAST) study. Korean J Intern Med 2015;30:460-70.

36. Shin TG, Choi JH, Jo IJ, et al. Extracorporeal cardiopulmonary resuscitation in patients with inhospital cardiac arrest: a comparison with conventional cardiopulmonary resuscitation. Crit Care Med 2011;39:1-7.

37. Korean Network for Organ Sharing (KONOS): Official Web site of the KONOS [Internet]; c2016 [cited 2016 July 1]. Available from: https:// www.konos.go.kr/konosis/index.jsp.

38. Krittayaphong R, Ariyachaipanich A. Heart Transplant in Asia. Heart Fail Clin 2015;11:563-72. 